\title{
Incidence and predictors of loss to follow up among HIV-infected adults at Pawi General Hospital, northwest Ethiopia: competing risk regression model
}

\author{
Moges Agazhe Assemie ${ }^{1}$, Kindie Fentahun Muchie ${ }^{2^{*}}\left[\right.$ and Tadesse Awoke Ayele ${ }^{2}$
}

\begin{abstract}
Objective: This study was aimed at assessing the incidence of lost-to-follow-up and its predictors among HIV-positive adults after initiation into antiretroviral therapy at Pawi General Hospital, northwest Ethiopia.

Results: The overall cumulative incidence of lost-to-follow-up after ART initiation was high, 11.6 (95\% Cl 9.8-13.7) per 100 adult-years follow-up time. Independent significant predictors of lost to follow up were being aged 15-28 years ( $a S H R=0.44 ; 95 \% \mathrm{Cl} 0.24-0.83$ ), being on WHO clinical stage IV (aSHR $=2.09 ; 95 \% \mathrm{Cl} 1.02-3.13$ ); and receiving isoniazid preventive therapy (aSHR $=0.11 ; 95 \% \mathrm{Cl} 0.06-0.18$ ).
\end{abstract}

Keywords: Lost to follow-up, Cumulative incidence, Competing risks regression, Sub-distribution model, Predictors, Associated factors, ART

\section{Introduction}

Globally, 17 million people were accessing antiretroviral therapy (ART), coverage being $46 \%$ at the end of 2015 [1]. In Ethiopia, nearly 1.2 million people were living with HIV/AIDS with an incidence of $1.2 \%$ [2]. At the end of 2013, the proportion of patients who ever started ART was $60.7 \%$, of whom $18.7 \%$ were lost-to-follow-up (LTFU) in Ethiopia [3].

Although ART significantly reduced mortality and improved life expectancy of HIV-infected patients, the attainment depends on regular patient follow-up [4]. Effective ART can control the virus and prevent transmission. A trial in 2011 showed that adherence to an effective ART reduced the risk of transmitting the virus to their uninfected sexual partner by $96 \%$ [5]. LTFU, undesirably blocks the immunological benefits of ART and increases AIDS-related hospitalizations, morbidity,

\footnotetext{
*Correspondence: mkindief@gmail.com

${ }^{2}$ Department of Epidemiology and Biostatistics, Institute of Public Health, College of Medicine and Health Sciences, University of Gondar, Gondar, Ethiopia

Full list of author information is available at the end of the article
}

and death in resource limited countries, has emerged as an everyday threat $[6,7]$. The incidence of LTFU was estimated to be 10.9 per 100 person-years in South Africa [8], 10 per 100 in Zambia [9], and 8.2 per 100 in Aksumnorthern Ethiopia [10].

The significant risk factors of LTFU are sex, youth, occupation, and lack of formal education [11-13] as well clinical characteristics, like CD4-count, WHO stage, functional status and Isoniazid Preventive Therapy (IPT) $[9,12,14-16]$.

The analysis of time-to-LTFU can be complicated by competing risks, deaths that alter the probability or completely preclude the occurrence of LTFU. This is distinct from censoring which merely prevents observing the time at which LTFU occurs [17-19]. In standard Kaplan-Meier analyses, those experiencing a competing event will be taken as censored. However, this is invalid because LTFU can no longer occur in those experiencing death, and such analyses will therefore overestimate the probability of the LTFU. Consequently, the method of Fine and Gray, competing-risks regression provides a 
useful alternative to Cox regression for time to event data in the presence of competing risks [18, 20,21].

There was limited evidence on the incidence of LTFU and its predictors in the study area. This study aimed to assess the incidence of LTFU and its predictors with death as a competing event among adult HIV positive patients who started ART at Pawi General Hospital, northwest Ethiopia.

\section{Main text \\ Methods}

An institution-based retrospective study was conducted at Pawi General Hospital. Pawi General Hospital is found in Pawi town of Benshangul Gumuz Regional State, northwest Ethiopia. The hospital has been providing chronic HIV care and support to both pre-ART and ART clients since 2005. Out of the 3210 patients enrolled for the care, 1760 had started ART in the hospital.

All HIV-infected adults who enrolled at Pawi General Hospital ART clinic in January 2012-December 2016 were considered for this study. To check sufficiency of samples, minimum sample size (583) was determined for the incidence and predictors using st power command of software Stata 14. Those who had at-least one follow-up visit were eligible to be included. We excluded those who had unknown initiation date, undefined outcome, and transferred in with incomplete base-line data minimizing important factors.

The data were collected using a standard data extraction sheet, entered using Epi Info version 7, and exported to statistical packages Stata version 14.0 for further analysis. The outcome variable in this study was time to LTFU (in months) with death as a competing event. Accordingly, participants were classified as either LTFU, died, or censored (transferred out or still under follow-up) at the end of the study period. 'LTFU' is defined as patients not taking ART refill for a period of 3 months or longer from the last attendance for refill and not yet classified as 'died' or 'transferred-out' [22]. 'Transferred out' are patients who were formally transferred out to another health facility. Besides, those patients who died and confirmed by physicians with the approval of the medical director of the hospital followed by recoded final outcome as death on the follow up chart were taken as died. Furthermore, a patient was taken as working functional status if a patient is able to perform usual work in or out of the house.

Incidence of LTFU was determined from the start of ART until the last follow-up visit or known date of death. We used competing risks regression with proportional sub-distribution hazard model to model cumulative incidence $[23,24]$. The model quantifies the instantaneous risk of failure from the LTFU and the overall impact of covariates on the incidence of LTFU [17], treating death as a competing risk $[18,23]$.

Variables in the bi-variable proportional sub-distribution hazard model with $\mathrm{p}$ value below 0.25 were subsequently included in the multivariable analysis. Schoenfeld residual and Cox Snell residual plot for the overall model fitness tests were used to check the model assumption. Results were taken as statistically significant for $\mathrm{p}<0.05$. Crude sub-hazard ratio (cSHR) and adjusted sub-hazard ratio (aSHR) with their respective 95\% confidence interval $(\mathrm{CI})$ were reported to show the strength of association.

\section{Results}

Six hundred two HIV infected adults were included in the final analysis. Of these, $4.2 \%$ died and $22.6 \%$ LTFU. More than half, 338 (56.2\%), of HIV positive adults were females. The median age at ART initiation was 32 years [IQR: 27-39]. About 189 (31.4\%) of the participants were aged 1528 years, whereas nearly half, 285 (47.3\%), were married. Furthermore, $270(44.8 \%)$ had no formal education, and only $2.5 \%$ had higher educational status (Table 1).

Nearly half, 313 (52\%) of the patients were eligible for ART by WHO clinical stage and total lymphocyte count, while 289 (48\%) were so by CD4 cell count criteria. The median baseline CD4 cell count was 217 (IQR: 119-341) cells $/ \mathrm{mm}^{3}$. Regarding nutritional status, 255 (42.4\%) of the participants were malnourished according to their body mass index (BMI). More than half, 325 (54.0\%), of the participants were initiated into ART at WHO stageIII. Cotrimoxazole Preventive Therapy (CPT) and IPT were given to 418 (69.4\%) and 340 (56.8\%), respectively.

Over two-thirds, 410 (68.1\%), of the patients started ART treatment with TDF-3TC-EFV and 36 (6\%) changed to another regimen within the first line, and 11 (2\%) switched to the second line. The majority of the participants, 444 (73.8\%), were at working functional status. Over one-fifth, 133 (22.1\%), had opportunistic infections, of whom 68 (11.3\%) had Tuberculosis, 49 (8.1\%) bacterial pneumonia and $16(2.7 \%)$ other opportunistic infections (Table 2).

The total follow-up time was 1175 adult-years observation with an incidence rate of 11.6 per 100 adult-years (95\% CI 9.8-13.7) of LTFU. The subjects were followed for a minimum of three and a maximum of 60 months after initiation into ART with 18 months median followup time. The median 'LTFU' time of 15 months for male was shorter than 23 months for female patients. Nearly half of the subjects were LTFU within the first 6 months and around $73 \%$ before a year. The difference between two estimates of cumulative probability of LTFU (one minus Kaplan-Meier and cumulative incidence in the 
Table 1 Baseline socio-demographic characteristics of HIV-positive adults on ART at Pawi General Hospital, northwest Ethiopia, Jan 2012-Dec 30, 2016

\begin{tabular}{|c|c|c|}
\hline Variables & $\begin{array}{l}\text { Frequency } \\
(n=602)\end{array}$ & Percent (\%) \\
\hline \multicolumn{3}{|l|}{ Age (years) } \\
\hline $15-28$ & 189 & 31.4 \\
\hline $29-34$ & 160 & 26.6 \\
\hline $35-44$ & 170 & 28.2 \\
\hline$>45$ & 83 & 13.8 \\
\hline \multicolumn{3}{|l|}{ Gender } \\
\hline Female & 338 & 56.2 \\
\hline Male & 264 & 43.8 \\
\hline \multicolumn{3}{|l|}{ Marital status } \\
\hline Single & 112 & 18.6 \\
\hline Married & 285 & 47.3 \\
\hline Divorced & 146 & 24.3 \\
\hline Widowed & 59 & 9.8 \\
\hline \multicolumn{3}{|l|}{ Educational status } \\
\hline No formal education & 270 & 44.8 \\
\hline Primary & 222 & 36.9 \\
\hline Secondary & 95 & 15.8 \\
\hline Higher & 15 & 2.5 \\
\hline \multicolumn{3}{|l|}{ Occupation } \\
\hline Daily labor & 150 & 24.9 \\
\hline Farmer & 200 & 33.2 \\
\hline Merchant & 67 & 11.1 \\
\hline Government employee & 48 & 8.0 \\
\hline Self-employee & 42 & 7.0 \\
\hline Student & 31 & 5.2 \\
\hline House wife & 64 & 10.6 \\
\hline
\end{tabular}

presence of death as a competing risk) is shown (Additional file 1). The additional file illustrated that the former overestimates the probability of experiencing LTFU at each follow-up time, except in the instance when there are no competing risks at the beginning of the follow up period.

The sub-hazard distribution proportionality assumption by schoenfeld residual global test was 0.4574, showed neither increasing nor decreasing pattern. As well, the Cox Snell residual plot almost overlay the Nelson-Aalen cumulative hazard (Additional file 2) showing the proportional sub-hazards assumption was satisfied.

Age, sex, CPT, WHO clinical stage, IPT, BMI, opportunistic infections, and functional status were fitted to the multivariable sub-distribution model. Accordingly, in the multivariable model adults in the over 45 years of age category lowered the sub-hazard ratio of LTFU by $56 \%$ $(\mathrm{aSHR}=0.44 ; 95 \% \mathrm{CI} 0.24-0.83)$ compared to those aged
Table 2 Baseline clinical characteristics of HIV-positive adults on ART at Pawi General Hospital, northwest Ethiopia, Jan 1, 2012-Dec 30, 2016

\begin{tabular}{|c|c|c|}
\hline Variables & $\begin{array}{l}\text { Frequency } \\
(\mathrm{N}=602)\end{array}$ & Percent (\%) \\
\hline \multicolumn{3}{|l|}{ IPT } \\
\hline Not received & 262 & 43.2 \\
\hline Received & 340 & 56.8 \\
\hline \multicolumn{3}{|l|}{ CPT } \\
\hline Not receiving & 184 & 30.6 \\
\hline Receiving & 418 & 69.4 \\
\hline \multicolumn{3}{|c|}{ Opportunistic infection } \\
\hline NoOl & 469 & 77.9 \\
\hline Have Ol & 133 & 22.1 \\
\hline \multicolumn{3}{|l|}{ WHO clinical stage } \\
\hline Stage I & 55 & 9.1 \\
\hline Stage II & 172 & 28.6 \\
\hline Stage III & 325 & 54.0 \\
\hline Stage IV & 50 & 8.3 \\
\hline \multicolumn{3}{|l|}{ ART regimen } \\
\hline $1 C(A Z T-3 T C-N V P)$ & 97 & 16.1 \\
\hline $1 \mathrm{~d}(\mathrm{AZT}-3 \mathrm{TC}-\mathrm{EFV})$ & 26 & 4.3 \\
\hline $1 \mathrm{e}(\mathrm{TDF}-3 \mathrm{TC}-\mathrm{EFV})$ & 410 & 68.1 \\
\hline 1 f(TDF-3TC-NVP) & 69 & 11.5 \\
\hline \multicolumn{3}{|l|}{ Functional status } \\
\hline Working & 444 & 73.8 \\
\hline Ambulatory & 99 & 16.4 \\
\hline Bedridden & 59 & 9.8 \\
\hline \multicolumn{3}{|l|}{ Adherence } \\
\hline Good & 585 & 97.2 \\
\hline Fair & 12 & 2.0 \\
\hline Poor & 5 & 0.8 \\
\hline \multicolumn{3}{|l|}{ Regimen change } \\
\hline No & 555 & 92.2 \\
\hline \multicolumn{3}{|l|}{ Yes } \\
\hline First line & 36 & 6.0 \\
\hline Second line & 11 & 1.8 \\
\hline \multicolumn{3}{|c|}{ Nutritional status (BMI) } \\
\hline Normal & 347 & 57.6 \\
\hline Malnourished & 255 & 42.4 \\
\hline \multicolumn{3}{|c|}{ CD4 cell count (cell/mm³) } \\
\hline$\leq 200$ & 360 & 43.2 \\
\hline$>200$ & 342 & 56.8 \\
\hline
\end{tabular}

AZT zidovudine, 3TC lamivudine, NVP nevirapine, EFV efavirenz, TDF tenofovier

15-28 years (Table 3). Adults who were on WHO clinical stage-IV increased the sub-hazard ratio of LTFU by 2.09 $(\mathrm{aSHR}=2.09 ; 95 \% \mathrm{CI} 1.19-3.67)$ times as compared to adults in stages-I and II. Being on IPT lowered the subdistribution hazard ratio for LTFU by $89 \%(\mathrm{aSHR}=0.11$; 95\% CI 0.06-0.18) as compared to their counter parts. 
Table 3 Multivariable competing risk regression analysis for predictors of LTFU among HIV-positive Adults at Pawi General Hospital, northwest Ethiopia, Jan 1, 2012-Dec 30, 2016

\begin{tabular}{|c|c|c|c|c|c|}
\hline \multirow[t]{2}{*}{ Variable } & \multicolumn{3}{|l|}{ Survival status } & \multirow[t]{2}{*}{ CSHR $[95 \% \mathrm{Cl}]$} & \multirow[t]{2}{*}{$\operatorname{aSHR}[95 \% \mathrm{Cl}]$} \\
\hline & Lost $(\mathrm{N}=136)$ & Death $(N=25)$ & Censored $(\mathrm{N}=441)$ & & \\
\hline \multicolumn{6}{|l|}{ Age (years) } \\
\hline $15-28$ & 49 & 6 & 134 & 1 & 1 \\
\hline $29-34$ & 34 & 9 & 117 & $0.72[0.47-1.11]$ & $0.76(0.50-1.16)$ \\
\hline $35-45$ & 41 & 5 & 124 & $0.86[0.57-1.3]$ & $0.85(0.56-1.29)$ \\
\hline$>45$ & 12 & 5 & 66 & $0.47(0.25-0.89)$ & $0.44(0.24-0.83)$ \\
\hline \multicolumn{6}{|l|}{ Sex } \\
\hline Female & 63 & 12 & 262 & 1 & 1 \\
\hline Male & 73 & 13 & 179 & $1.60(1.14-2.23)$ & $1.40(0.99-1.99)$ \\
\hline \multicolumn{6}{|l|}{ Nutritional status } \\
\hline Normal & 69 & 10 & 268 & 1 & 1 \\
\hline Malnourished & 67 & 15 & 173 & $1.38(0.99-1.93)$ & $0.73(0.52-1.02)$ \\
\hline \multicolumn{6}{|c|}{ WHO clinical stage } \\
\hline Stage $(I / I I)$ & 36 & 5 & 186 & 1 & 1 \\
\hline Stage III & 79 & 13 & 232 & $1.55(1.05-2.29)$ & $1.34(0.92-1.95)$ \\
\hline Stage IV & 21 & 7 & 23 & $2.77(1.62-4.76)$ & $2.09(1.19-3.67)$ \\
\hline \multicolumn{6}{|l|}{ Functional status } \\
\hline Asymptomatic & 85 & 6 & 353 & 1 & 1 \\
\hline Symptomatic & 51 & 19 & 88 & $1.63(1.16-2.29)$ & $1.03(0.72-1.45)$ \\
\hline \multicolumn{6}{|l|}{ Isoniazid } \\
\hline Not received & 115 & 20 & 125 & 1 & 1 \\
\hline Received & 21 & 5 & 316 & $0.11(0.07-0.17)$ & $0.11(0.07-0.18)$ \\
\hline \multicolumn{6}{|l|}{ CPT } \\
\hline Not received & 46 & 12 & 126 & 1 & 1 \\
\hline Received & 90 & 13 & 315 & $0.79(0.56-1.12)$ & $0.84(0.59-1.20)$ \\
\hline \multicolumn{6}{|c|}{ Opportunistic infection } \\
\hline No & 93 & 13 & 363 & 1 & 1 \\
\hline Yes & 43 & 12 & 77 & $1.58(1.12-2.23)$ & $0.91(0.63-1.30)$ \\
\hline
\end{tabular}

\section{Discussion}

Several studies have shown that LTFU poses challenges to the successful implementation of ART programs in low resource settings [25]. This study aimed to assess the incidence of LTFU and its predictors among HIV positive adults after they were initiated into ART.

The incidence estimated in this study, 11.6 per 100 adult-years was consistent with 12.1-15.3 person-year reported in Latin and Caribbean countries, and 10.9 in South Africa [26, 27]. However, the incidence estimated was higher than those of studies in Zambia [9], and Aksum (northern-Ethiopia) [10]. The variation might be explained by differences in the study settings, transfer out, health seeking behavior, and late initiation of the community into ART at advanced WHO clinical
stages-III and IV. The high rate of transfer out without prior information to their original health institution of initial registration necessary for proper recording may be another reason.

The proportion of LTFU of $22.6 \%$ seen in this study was lower than $36.6 \%$ reported in Cameroon [13] and $27.6 \%$ in South Africa [27]. It was, however, higher than the 15.5,17.7, 15.6, 14.5, 19.6, and $21.7 \%$ detected in India, Sub-Saharan Africa, Nigeria, Hadiya (SouthernEthiopia), Ethiopian public hospitals, and in Southern Nations Nationalities and Peoples (SNNP) Region (Ethiopia), respectively [11-13, 28-30]. The LTFU in our study turned out to be higher than those of the preceding reports because of travel costs patients had to cover to reach clinics. That means our study area is relatively 
under developed and sparsely populated with limitedinfrastructure and resources which are directly or indirectly related to health service delivery and access.

The findings of the multivariable sub-distribution hazard regression revealed that, age category (1528 years), not being on IPT prophylaxis, and WHO clinical stage-IV were independent predictors of LTFU. The finding implied that as age increased the probability of LTFU decreased. Patients aged above 45-years had lower risk of LTFU as compared to those aged 1528 years. This finding was consistent with those of studies conducted in Southern Nigeria, South Africa, Oromia and SNNP Region in Ethiopia [28, 31-34]. This could be due to immaturity in analytical thinking, and particular challenges associated with puberty. Fear of stigma and discrimination, as this younger age group is dependent on others, and independent members of this age group could be more mobile as compared to the older population [12]. This idea is also congruent with the qualitative findings indicating that the younger age groups who fear stigmatization are more prone to being LTFU from ART [35]. The finding is different from that of a study conducted in Nigeria [11] which showed younger groups were at a lower risk of LTFU.

HIV-positive adults in advanced clinical stage-IV increased the risk of LTFU by two-fold as compared to those in WHO clinical stage-I/II. This evidence was supported by studies conducted in South Africa, Uganda, India and Oromia (Ethiopia) [8, 12, 15, 36]. This is because more LTFU could be due to death and patients at advanced clinical stage are more likely to die because of side effects of ART within the first 6 months of ART initiation [37]. Beside severely ill patients might unable to revisit the service rendered. Another study in Nigeria indicated that half of the participants were in immunosuppressed advanced stages-III and IV [11].

Moreover, those who received IPT had a lower risk of LTFU as compared to those did not. This result is consistent with studies conducted in southern and northern Ethiopia, and Oromia (Ethiopia) [16, 31, 33]. The IPT prophylaxis recommended by national ART guidelines to prevent the occurrence of Tuberculosis co-infection, one of the causes of morbidity and mortality, might have been directly or indirectly imposed on LTFU.

\section{Conclusions}

The incidence of LTFU was higher than in most studies in Ethiopia. HIV positive patients aged 15-28 years, WHO clinical stage-IV, and not being on IPT prophylaxes are at higher risk for LTFU. Those patients need support in terms of reminders, surveillance, and tracing mechanism to reduce LTFU.

\section{Limitations}

Those persons who had no complete base line data were not included. Predictors like viral load, hemoglobin test, and clinical factors were not included as they were not well documented on patient cards. Thus, this finding should be interpreted with this limitation in mind.

\section{Additional files}

Additional file 1. Kaplan-Meier failure curve and cumulative incidence function for Lost to follow up.

Additional file 2. Cox Snell residual plot for overall fitness of the model.

\section{Authors' contributions}

All authors equally participated in the design, conception, analysis and interpretation. MAA, KFM, and TAA facilitated the data collection and management, drafted, and critically reviewed the manuscript. All authors read and approved of the final manuscript.

\section{Author details}

${ }^{1}$ Department of Public Health, College of Health Sciences, Debre Markos University, Debre Markos, Ethiopia. ${ }^{2}$ Department of Epidemiology and Biostatistics, Institute of Public Health, College of Medicine and Health Sciences, University of Gondar, Gondar, Ethiopia.

\section{Acknowledgements}

The authors would like to express their deepest gratitude to data collectors. We would like to extend our gratitude to Pawi General Hospital administrators for their cooperation.

\section{Competing interests}

The authors declare that they have no competing interests.

\section{Availability of data and materials}

The datasets used and/or analyzed during the current study are available from the corresponding author on reasonable request.

\section{Consent for publication}

Not applicable.

\section{Ethics approval and consent to participate}

Ethical clearance was obtained from the Institutional Review Board of the Institute of Public Health, University of Gondar. Written permission was obtained from hospital administrative bodies. Data were anonymized and handled confidentially during all phases of research activities.

\section{Funding}

Not applicable.

\section{Publisher's Note}

Springer Nature remains neutral with regard to jurisdictional claims in published maps and institutional affiliations.

Received: 16 October 2017 Accepted: 7 May 2018

Published online: 10 May 2018

References

1. WHO. US/HIV AIDS report. 2015

2. UNAIDS. HIV/AIDS report. 2014.

3. HAPCO. Federal Democratic Republic of Ethiopia, HIV/AIDS Prevention and Control Office. 2014 
4. Cohen MS, Chen YQ, McCauley M, Gamble T, Hosseinipour MC, Kumarasamy $\mathrm{N}$, et al. Prevention of HIV-1 infection with early antiretroviral therapy. N Engl J Med. 2011;365(6):493-505.

5. World Health Organization. HIV/AIDS fact sheet. Geneva: WHO; 2017.

6. Estill J, Tweya H, Egger M, Wandeler G, Feldacker C, Johnson LF, et al. Tracing of patients lost to follow-up and HIV transmission: mathematical modelling study based on two large ART programmes in Malawi. J Acquir Immune Defic Syndr (1999). 2014;65(5):e179

7. Taiwo B. Understanding transmitted HIV resistance through the experience in the USA. Int J Infect Dis. 2009;13(5):552-9.

8. Mberi MN, Kuonza LR, Dube NM, Nattey C, Manda S, Summers R. Determinants of loss to follow-up in patients on antiretroviral treatment, South Africa, 2004-2012: a cohort study. BMC Health Serv Res. 2015;15(1):259.

9. Li MS, Musonda P, Gartland M, Mulenga PL, Mwango A, Stringer JS, et al. Predictors of patient attrition according to different definitions for loss to follow-up: a comparative analysis from Lusaka, Zambia. J Acquir Immune Defic Syndr (1999). 2013;63(3):e116.

10. Tadesse K, Fisiha H. Predictors of loss to follow up of patients enrolled on antiretroviral therapy: a retrospective cohort study. J AIDS Clin Res. 2014;5(393):2

11. Eguzo KN, Lawal AK, Eseigbe CE, Umezurike CC. Determinants of mortality among adult HIV-infected patients on antiretroviral therapy in a rural hospital in Southeastern Nigeria: a 5-year cohort study. AIDS Res Treat. 2014;2014:6.

12. Alvarez-Uria G, Naik PK, Pakam R, Midde M. Factors associated with attrition, mortality, and loss to follow up after antiretroviral therapy initiation: data from an HIV cohort study in India. Glob Health Action. 2013;6(1):21682

13. Bekolo CE, Webster J, Batenganya M, Sume GE, Kollo B. Trends in mortality and loss to follow-up in HIV care at the Nkongsamba Regional hospital, Cameroon. BMC Res Notes. 2013;6(1):512.

14. Palombi L, Marazzi MC, Guidotti G, Germano P, Buonomo E, Scarcella $P$, et al. Incidence and predictors of death, retention, and switch to second-line regimens in antiretroviral-treated patients in sub-Saharan African Sites with comprehensive monitoring availability. Clin Infect Dis. 2009;48(1):115-22.

15. Assefa $Y$, Lynen L, Kloos H, Hill P, Rasschaert F, Hailemariam D, et al. Brief report: long-term Outcomes and their determinants in patients on antiretroviral treatment in Ethiopia, 2005/6-2011/12: a retrospective cohort study. JAIDS J Acquir Immune Defic Syndr. 2015:70(4):414-9.

16. Dessalegn $M$, Tsadik $M$, Lemma H. Predictors of lost to follow up to antiretroviral therapy in primary public hospital of Wukro, Tigray, Ethiopia: a case control study. J AIDS HIV Res. 2015;7(1):1-9.

17. Koller MT, Raatz H, Steyerberg EW, Wolbers M. Competing risks and the clinical community: irrelevance or ignorance? Stat Med. 2012;31(11-12):1089-97.

18. Fine JP, Gray RJ. A proportional hazards model for the subdistribution of a competing risk. J Am Stat Assoc. 1999;94(446):496-509.

19. Lau B, Cole SR, Gange SJ. Competing risk regression models for epidemiologic data. Am J Epidemiol. 2009;170(2):244-56.

20. Cox D. Regression models and life tables. J Roy Stat Soc Ser B. 1972;34(2):187-220.

21. Satagopan J, Ben-Porat $L$, Berwick M, Robson M, Kutler D, Auerbach A. A note on competing risks in survival data analysis. Br J Cancer. 2004;91(7):1229.

22. Chi BH, Yiannoutsos CT, Westfall AO, Newman JE, Zhou J, Cesar C, et al. Universal definition of loss to follow-up in HIV treatment programs: a statistical analysis of 111 facilities in Africa, Asia, and Latin America. PLoS Med. 2011;8(10):e1001111.

23. Bakoyannis $\mathrm{G}$, Touloumi $\mathrm{G}$. Practical methods for competing risks data: a review. Stat Methods Med Res. 2012;21(3):257-72.

24. Gooley TA, Leisenring W, Crowley J, Storer BE. Estimation of failure probabilities in the presence of competing risks: new representations of old estimators. Stat Med. 1999;18(6):695-706.

25. Rosen S, Fox MP, Gill CJ. Patient retention in antiretroviral therapy programs in sub-Saharan Africa: a systematic review. PLoS Med. 2007;4(10):e298.

26. Carriquiry G, Fink V, Koethe JR, Giganti MJ, Jayathilake K, Blevins M, et al. Mortality and loss to follow-up among HIV-infected persons on longterm antiretroviral therapy in Latin America and the Caribbean. J Int AIDS Soc. 2015;18(1):20016.

27. Dalal RP, MacPhail C, Mqhayi M, Wing J, Feldman C, Chersich MF, et al. Characteristics and outcomes of adult patients lost to follow-up at an antiretroviral treatment clinic in Johannesburg, South Africa. JAIDS J Acquir Immune Defic Syndr. 2008;47(1):101-7.

28. Teshome W, Belayneh M, Moges M, Mekonnen E, Endrias M, Ayele S, et al. Do loss to follow-up and death rates from ART care vary across primary health care facilities and hospitals in south Ethiopia? A retrospective follow-up study. HIV/AIDS (Auckland, NZ). 2015;7:167-74.

29. Wilhelmson S, Reepalu A, Tolera Balcha T, Jarso G, Björkman P. Retention in care among HIV-positive patients initiating second-line antiretroviral therapy: a retrospective study from an Ethiopian public hospital clinic. Glob Health Action. 2016;9(1):29943.

30. Ayele W, Mulugeta A, Desta A, Rabito FA. Treatment outcomes and their determinants in HIV patients on anti-retroviral treatment program in selected health facilities of Kembata and Hadiya zones, Southern Nations, Nationalities and Peoples Region, Ethiopia. BMC Public Health. 2015;15(1):826.

31. Shargie EB, Lindtjørn B. Determinants of treatment adherence among smear-positive pulmonary tuberculosis patients in Southern Ethiopia. PLoS Med. 2007:4(2):e37.

32. Wang B, Losina E, Stark R, Munro A, Walensky RP, Wilke M, et al. Loss to follow-up in a community clinic in South Africa: roles of gender, pregnancy and CD4 count. SAMJ S Afr Med J. 2011;101(4):253-7.

33. Megerso A, Garoma S, Tolosa Eticha TW, Daba S, Tarekegn M, Habtamu Z. Predictors of loss to follow-up in antiretroviral treatment for adult patients in the Oromia region, Ethiopia. HIV/AIDS (Auckland, NZ). 2016:8:83.

34. Meloni ST, Chang C, Chaplin B, Rawizza H, Jolayemi O, Banigbe B, et al. Time-dependent predictors of loss to follow-up in a large HIV treatment cohort in Nigeria. Open Forum Infect Dis. 2014;1:ofu055.

35. Wubshet M, Berhane Y, Worku A, Kebede Y. Death and seeking alternative therapy largely accounted for lost to follow-up of patients on ART in northwest Ethiopia: a community tracking survey. PLOS One. 2013;8(3):e59197.

36. Nakiwogga-Muwanga A, Alamo-Talisuna S, Musaazi J, Kambugu A, Ssekawungu P, Katabira E, et al. Inadequate monitoring in advanced stages of disease with lack of supportive counseling increases attrition among patients on antiretroviral treatment at a large urban clinic in Uganda. J Int Assoc Provid AIDS Care (JIAPAC). 2014;13(6):547-54.

37. Bhatta L, Klouman E, Deuba K, Shrestha R, Karki DK, Ekstrom AM, et al. Survival on antiretroviral treatment among adult HIV-infected patients in Nepal: a retrospective cohort study in far-western Region, 2006-2011. BMC infectious diseases. 2013;13(1):604 